\title{
PRESSURE INVESTIGATION OF TUNNELING IN AN InAlAs-InGaAs DOUBLE BARRIER STRUCTURE
}

\author{
L.A. CURY, \\ INSA-CNRS, 31077 Toulouse and SNCI-CNRS, 38047 Grenoble, France
}

L.H. DMowskI,

High Pressure Research Center "Unipress", Sokolowska 29, 01-142 Warszawa, Poland
A. Celeste, J.C. Portal,

INSA-CNRS, 31077 Toulouse and SNCI-CNRS, 38047 Grenoble, France

\section{Davies, M. Heath,}

Department of Physics, University of Nottingham, Nottingham NG7 2RD, U.K.

$$
\text { D.L. Sivco AND A.Y. Cho }
$$

AT \& T Bell Laboratories, Murray Hill, New Jersey 07974, USA

(Received August 8, 1990)

\begin{abstract}
An Ino.52 $\mathrm{Al}_{0.48} \mathrm{As}-\mathrm{In}_{0.53} \mathrm{Ga}_{0.47} \mathrm{As}$ double-barrier structure was studied under hydrostatic pressures up to $15 \mathrm{kbar}$. Two resonances in the $I(V)$ curves are observed. The possible tunneling mechanisms to interpret this effect are analysed.
\end{abstract}

PACS numbers: $07.35 .+\mathrm{k}, 73.20 . \mathrm{Dx}, 79.80 .+\mathrm{w}$

IIydrostatic pressure as a tool to study the $\Gamma-X$ band mixing in double-barrier structures based on III-V compounds has attracted much attention recently [1-3]. The effect of an applied pressure on the band structure of these materials is to produce a variation on the $X$ - and $L$-conduction band minima relative to the $\Gamma$-minimum. We have investigated an asymetric $\mathrm{In}_{0.52} \mathrm{Al}_{0.48} \mathrm{As}-\mathrm{In}_{0.53} \mathrm{Ga}_{0.47} \mathrm{As}$ 
double-barrier structure under hydrostatic pressures up to $15 \mathrm{kbar}$. The studied sample has two $\mathrm{In}_{0.52} \mathrm{Al}_{0.48} \mathrm{As}$ barriers with different widths, $40 \AA$ and $70 \AA$. The enclosed well between them has $60 \AA$ width. The complete structure, in order of growth from the $n^{+} \operatorname{InP}(100)$ substrat, is: (i) $0.5 \mu$ m of $n=2 \times 10^{18} \mathrm{~cm}^{-3} \mathrm{InGaAs}$; (ii) $0.5 \mu \mathrm{m}$ of $n=2 \times 10^{18}$ to $2 \times 10^{16} \mathrm{~cm}^{-3}$ InGaAs; (iii) $250 \AA$ of $n=2 \times 10^{16} \mathrm{~cm}^{-3}$ InGaAs; (iv) $70 \AA$ of undoped InAlAs; (v) $60 \AA$ of undoped InGaAs; (vi) $40 \AA$ of undoped InAlAs; (vii) $250 \AA$ of $n=2 \times 10^{16} \mathrm{~cm}^{-3} \mathrm{InGaAs}$; (viii) $0.5 \mu \mathrm{m}$ of $n=2 \times 10^{16}$ to $2 \times 10^{18} \mathrm{~cm}^{-3}$ InGaAs; (ix) $0.5 \mu \mathrm{m}$ of $n=2 \times 10^{18} \mathrm{~cm}^{-3} \mathrm{InGaAs}$, top contact. $50 \mu \mathrm{m}$ mesas were fabricated using standard litography technique.
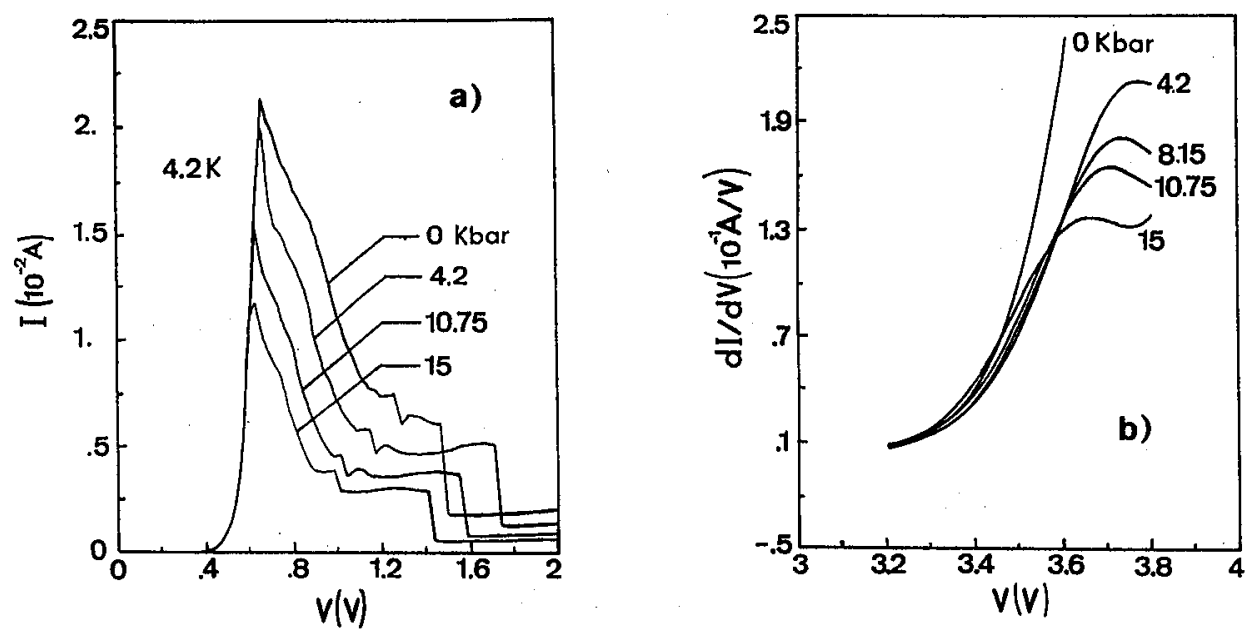

Fig. 1. Current-voltage characteristics for the first resonance (a) and conductance $(\mathrm{d} I / \mathrm{d} V$ ) versus voltage for the second resonance (b) measured at $4.2 \mathrm{~K}$ for different hydrostatic pressures.

The calculation of the transmission coefficient for the square double-barrier, utilizing the band structure parameters for In AlAs and InGaAs and including mass non-parabolicity correction, gives two peaks corresponding to two different energy levels in the well: $\epsilon_{1}=102 \mathrm{meV}$ and $\epsilon_{2}=336 \mathrm{meV}$.

The $I(V)$ characteristics of our sample at atmospheric pressure present a first pronounced peak at $V_{1}=0.68 \mathrm{~V}$ (see Fig. 1(a)). At this pressure, up to $3.8 \mathrm{~V}$, it was not possible to define the voltage position for the inflexion point corresponding to the second resonance. Investigations at voltages higher than $3.8 \mathrm{~V}$ (note the high current associated) could degrade the sample. This position, $V_{2}=3.827 \mathrm{~V}$, was obtained by extrapolation from the data for higher pressures. It is worth to mention at this point, that the voltage accross the double-barrier region of the structure represents only a fraction of the total applied voltage. Indeed, a large amount of this voltage is dropped in the lightly doped contact regions on both sides of the structure.

A reduction of the first resonance current peak is well understcod. As the 

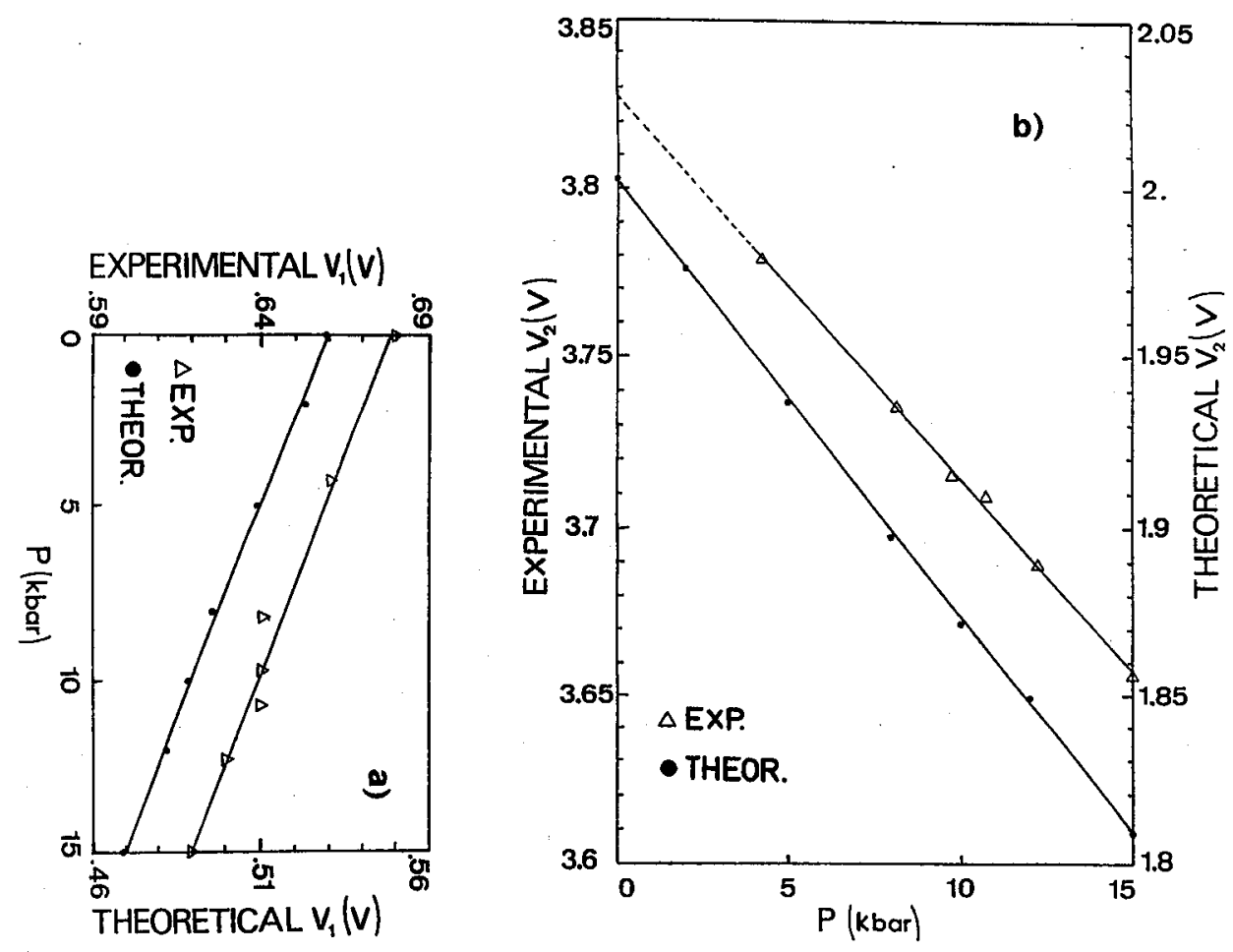

Fig.2. Experimental (maximum of conductance) and theoretical (maximum of transmission coefficient) data for the first (a) and second (b) resonances as a function of pressure, $P$. The experimental slopes are: $-3.8 \mathrm{meV} / \mathrm{kbar}$ and $-11.4 \mathrm{meV} / \mathrm{kbar}$ while the theoretical ones are: $-4.0 \mathrm{meV} / \mathrm{kbar}$ and $-12.9 \mathrm{meV} / \mathrm{kbar}$.

pressure $P$ increases, the effective masses increase, thus leading to a decrease of the transmission coeflicient peak and consequently, to a decrease of the current. Its position also shifts down to lower voltages, because the energy of the quasi-bound state in the well becomes smaller as the effective mass increases. For the second resonance, at higher voltages, the same pressure effects are observed (see Fig. 1(b)). The current amplitude at the inflexion point is also reduced but a more pronounced differential conductance peak is obtained as $P$ is increased. The second resonance is much broader than the first one. As the mean-width $\Delta$ is inversely proportional to the dwell time in the state, the latter is smaller for the second resonance and the electron escapes more rapidely. The displacement to lower voltages of the differential conductance peak for the second resonance, due to the pressure, is also observed.

We would like to stress, that we can not relate this weak resonant structure, at higher voltages, to a transmission of incident electron through the resonant state in the $X$-valley minimum as done by Mendez and co-workers for GaAs-AlGaAs 
system [3]. In our system tunneling processes through a resonant state in the $X$-valley is not possible due to the too high energy above the InAlAs $\Gamma$-conduction band minimum.

Tunneling via states in the $L$-minimum, although possible (as remarked by Mendez and co-workers [3]), would imply the violation of the transverse wave-vector conservation law, what is not likely to happen in our system, due to the short dwell time in the well which does not allow for enough scattering events.

We believe that the tunneling processes in the studied structure are quite controlled by $\Gamma$-like confining potentials and both observed NDC structures are related to the two resonant $\Gamma$-like levels in the InGaAs well. The experimental voltage position of the first and second resonances as well as voltage positions of the transmission coefficient peaks, calculated theoretically for a given incident energy $(10 \mathrm{meV})$ are plotted versus pressure in Fig. 2. The theoretical slopes of the curves do not depend on the energy of the incident electron. In the calculations we have considered the transverse wave vector conservation and the $\Gamma$-like confining potential only. We have not taken into account the electric field effects caused by the space charge in the emitter accumulation layer, nor the voltage drop in the gradual doping layers. However, we obtained qualitatively well the same linear behaviour of the voltage peaks for the resonances as a function of pressure.

\section{References}

[1] T.J. Foster, M.L. Leadbeater, D.K. Maude, E.S. Alves, L. Eaves, M. Henini, O.H. Hughes, A. Celeste, J.C. Portal, D. Lancefield, A.R. Adams, Solid-State Electron. 32, 1731 (1989).

[2] E.E. Mendez, L.L. Chang, Surf. Sci. 229, 173 (1990).

[3] E.E. Mendez, E. Calleja, C.E.T. Goncalves da Silva, L.L. Chang, W.I. Wang, Phys. Rev. B 33, 7368 (1986). 